\title{
A Diet and Exercise Intervention during Chemotherapy for Breast Cancer
}

\author{
Zora Djuric $^{*}, 1$, Jennifer S. Ellsworth ${ }^{1}$, Anne L. Weldon ${ }^{1}$, Jianwei Ren ${ }^{1}$, Caroline R. Richardson ${ }^{1}$, \\ Kenneth Resnicow ${ }^{2}$, Lisa A. Newman ${ }^{3}$, Daniel F. Hayes ${ }^{4}$ and Ananda Sen ${ }^{1}$ \\ ${ }^{1}$ Departments of Family Medicine, ${ }^{2}$ Health Behavior and Health Education, ${ }^{3}$ Surgery and ${ }^{4}$ Internal Medicine, University \\ of Michigan, Ann Arbor, MI 48109, USA
}

\begin{abstract}
Weight gain is an important concern that impacts on breast cancer outcomes and general health in survivorship. This randomized, pilot study evaluated whether or not women could comply with a weight control program that is initiated at the beginning of chemotherapy for breast cancer. The program sought to prevent weight gain using a low-fat, high fruit-vegetable diet combined with moderate physical activity. The intervention was implemented using a telephone counseling approach that blended motivational interviewing with social cognitive theory. A total of 40 women were recruited over 9 months at the University of Michigan Comprehensive Cancer Center. This represents 55\% of eligible women referred to the study and indicates that interest in a healthy lifestyle program at the initiation of chemotherapy for breast cancer was high. Subjects who dropped out had significantly lower fruit and vegetable intakes and lower blood carotenoids at baseline than subjects who completed the study. Statistically significant beneficial effects were observed on fruit and vegetable intakes, physical activity and breast cancer-specific well-being by the intervention. Mean body fat from dual energy X-ray absorptiometry increased in the written materials arm and decreased in the intervention arm. Of the enrolled women, $75 \%$ completed 12 months on study and satisfaction with study participation was high. These data indicate that lifestyle intervention during breast cancer treatment is feasible during treatment with chemotherapy for breast cancer and benefits women in several domains.
\end{abstract}

Keywords: Obesity, breast cancer, diet, physical activity, weight gain.

\section{INTRODUCTION}

Breast cancer is the most common cancer in women, and as treatments have improved the number of breast cancer survivors has grown [1]. Weight gain is an important concern that impacts on cancer outcomes and general health in survivorship. Recurrence rates and survival in early-stage disease have been shown to be adversely affected by increased body weight in most studies [2-4]. In a recent cohort study, every $5 \mathrm{~kg}$ weight gain post a breast cancer diagnosis increased cardiovascular (19\%) and breast cancer specific (13\%) mortality [5]. The weight gain in breast cancer survivors may stem from decreased activity, increased caloric intake and/or obesogenic medications such as steroids, and it is unlikely to be lost after treatment has ended $[2,6]$. Interestingly, women in the normal body mass index (BMI) range have been shown to be at greater risk for weight gain through treatment than heavier women [7].

Although not all studies have shown significant weight gain in breast cancer survivors, an increase in body fat has been consistent in studies that examined body composition during chemotherapy $[3,8-11]$. Although the significance of body fat gain in breast cancer patients is not yet known, body fat accumulation is a major health concern in the population in general. In postmenopausal women not taking hormone replacement therapy, breast cancer risk was more strongly associated with body fat than BMI (RR 3.41 for the top quintile of body fat) [12].

*Address correspondence to this author at the University of Michigan, 1500 E. Hospital Drive, Room 2150 Cancer Center, Ann Arbor, MI 48109-5930, USA; Tel: 734-615-6210; Fax: 734-647-9817; E-mail: zoralong@umich.edu
Weight gain can be prevented by lifestyles that include both a prudent diet and exercise [13, 14]. In the Nurse's Health Study, the largest weight gains over time were in women who changed from a prudent diet to a Western diet (5-6.8 $\mathrm{kg}$ over 4 years) [14]. Many epidemiological studies have shown that healthy eating patterns are associated with weight control and protect against diabetes and cardiovascular disease risks [15-17]. Prevention of weight gain in breast cancer survivors therefore should have significant beneficial effects on not only recurrence risks but health concerns that are more common than recurrence such as risks of cardiovascular diseases and diabetes, and even quality of life [18-20]. Finding effective weight control interventions for breast cancer patients therefore seems especially important.

In breast cancer survivors, diet quality may also affect disease recurrence. The Women's Intervention Nutrition Study showed that a low-fat diet, which resulted a in $2.7 \mathrm{~kg}$ weight loss, prevented recurrence by $24 \%$ in breast cancer survivors after a median follow up of 5 years [21]. A low-fat, high fruit-vegetable intervention in the Women's Healthy Eating and Living Study did not prevent recurrence, nor affect weight change; however, women who at baseline both consumed at least 5 servings/day of fruits and vegetables and exercised the equivalent of at least 30 minutes/day, 6 days/week, at study entry had $44 \%$ lower recurrence rates [22].

Associations of increased physical activity and reduced breast cancer risk have been consistent across studies [3]. Physical activity is important for preventing weight gain, and 
a goal of $30 \mathrm{~min} /$ day should be effective and achievable (unlike for preventing weight regain after a loss, which may require an hour or more per day) [23-26]. In breast cancer patients receiving treatment, most published physical activity interventions have been small, and limited in length, not coinciding with the entire length of medical treatment [2729]. Nonetheless, beneficial effects were noted on fitness, body weight, and various aspects of quality of life, including fatigue [30-34]. Conversely, weight gain is known to decrease quality of life $[35,36]$.

We conducted a randomized, pilot study to evaluate whether or not women could comply with a weight control program that is initiated during chemotherapy for breast cancer. The program sought to prevent a gain in body fat using a low-fat, high fruit-vegetable diet combined with moderate physical activity. The primary goals of the study were to evaluate feasibility of recruiting women early in their breast cancer treatment and to obtain estimates of changes in body fat and weight over one year. Secondary outcomes were psycho-social well-being and compliance to physical activity and dietary goals.

\section{METHODS}

\section{Subject Eligibility and Recruitment}

Women over age 18 with a stage I-IIIA breast cancer diagnosis and with BMI values of $20-45 \mathrm{~kg} / \mathrm{m}^{2}$ were eligible for participation. In addition, eligible subjects were either scheduled for chemotherapy treatment or were within two weeks of starting chemotherapy. Other eligibility criteria included stable body weight within 5 pounds in the past 2 months, physician approval for participating in a weight control program that includes recommendations for exercise and a diet low in fat and high in fruits and vegetables, and willing and able to follow advice for exercise and diet quality.

Exclusion criteria included having: a recurrence, a second primary tumor or other history of malignant tumors, previous chemotherapy for cancer within the past 10 years (with the exception of those who have currently completed no more than two weeks of chemotherapy), or previous hormonal therapy for cancer within the past 5 years, following a medically-prescribed diet, currently participating in a formal weight loss program, or having medical conditions that preclude safe exercise.

Potential subjects for the study were identified by their clinical care providers. This was facilitated by a weekly meeting of clinicians and researchers that reviewed eligibility of patients for clinical trials. If a patient was potentially eligible, the clinician presented the study to the patient. Patients who agreed to be contacted about the study were subsequently called by the study coordinator. Logs of eligibility screening were kept to assess recruitment success rates to this study, and the total numbers of new breast cancer patients were tracked by the research nurse from the Breast Care Clinic. Recruitment spanned January through September of 2008 and the study was approved by the University of Michigan Institutional Review Board (HUM000012524, Clinical Trials registration
NCT00583726). Subjects gave written, informed consent to participate in the study.

\section{Assessments}

All study participants were asked to complete assessment visits three times: at baseline, six months and twelve months. Subjects were paid $\$ 25$ for each study visit. At these visits, subjects were weighed, provided a blood sample, and filled out questionnaires. Fasting 10-ml blood samples were drawn for preparation of serum, whenever possible, at each visit. The samples were taken from a peripheral arm vein unless the subject had in a stable port that she wanted used. The baseline and 12 month visits also included measures of body composition by dual-energy X-ray absorptiometry (DXA).

Anthropometric measures were made using standardized techniques. These included measures of height, weight, blood pressure, waist and hip circumference, and body fat. Height was measured without shoes while standing on a level, hard surface with a stadiometer. Weight was measured in light clothing to the nearest quarter pound with a Professional Beam Scale. The blood pressure reading by auscultation was obtained contralateral to the side of breast cancer surgery whenever possible. Waist and hip circumference measures were obtained without restrictive garments to the nearest $0.1 \mathrm{~cm}$ with a flexible tape measure. Waist circumference was measured mid-point between the bottom rib and hip bone, keeping the tape in contact with the curve of the back. Hip circumference was obtained at the widest point of the hip. Percent body fat was measured from whole body scans by DXA using a GE Lunar Prodigy ADVANCE Plus instrument (GE Healthcare Inc.). For two subjects who did not fit within the DXA field, scans were obtained for each half of the body and averaged.

There were several questionnaires administered. The demographic and health questionnaire captured age, race/ ethnicity, education, household characteristics, health status and medication use. Health status and medication use were included in a Health Update questionnaire given at 6 and 12 months. Physical activity was assessed using a validated questionnaire from the Women's Health Initiative [37]. This questionnaire captures time spent walking at various speeds and performing mild, moderate and strenuous activities. The Functional Assessment of Cancer Therapy (FACT-B) wellbeing questionnaire included physical, emotional, functional, social, anemia, fatigue and breast cancer-specific quality of life domains, and this was developed specifically for breast cancer patients $[38,39]$. The FACT-G consists of only the physical, emotional, functional, social domains. Self-efficacy and self-confidence for maintaining a healthy lifestyle were measured using 6 items for each domain and a 5-point Likert-type scale. Finally, at the 12-month visit, all subjects were asked to evaluate the study using a 7-item questionnaire, and women in the telephone arm were asked to evaluate the counseling using a 9-item questionnaire. Diagnosis and treatment information was abstracted from patient medical charts.

Dietary intakes were assessed at each study visit by dietary screeners at 0,6 and 12 months (19-item All Day Screener for fruit and vegetables and the 17-item Percent Energy from Fat Screener) and by one un-announced 24- 
hour recall. The screeners were initially developed by the National Cancer Institute [40-42]. Dietary recalls were obtained by the research dietitian associated with the study at the baseline visit and by a clinical research dietitian not associated with the study before the 6 and 12 month study visits. The recalls were completed using a modified USDA five-pass method [43, 44]. The recalls were analyzed for nutrient intakes, fruit and vegetable servings and overall diet quality index using the Nutrition Data System Research Software (University of Minnesota, Nutrition Coordinating Center, database and software version 2008).

\section{Blood Measures}

Assays for cholesterol, HDL, and triacylglycerol (triglycerides) were done using a Cobas Mira Chemistry analyzer from Roche Diagnostics Corporation (Indianapolis, IN). LDL was calculated from the Friedewald equation [45]. High sensitivity C-reactive protein (CRP) was measured using a latex immunoturbimetric assay. Values above 10 $\mathrm{mg} / \mathrm{ml}$ for CRP were excluded as a possible indication of infection or other acute inflammatory condition: this criterion excluded 6 of 191 samples. C-peptide (insulin), insulin-like growth factor 1 (IGF-1) and its binding protein 3 (IGF-bp3) were assayed using the Immulite chemiluminescent assay system from Diagnostic Products Corporation (Los Angeles, CA). Glucose was measured with a hexokinase colorimetric assay. These assays were done by the Michigan Diabetes Research and Training Center Core Chemistry Laboratory. The facility calibrates all assays with standards and analyzes quality control samples daily.

For analysis of fat-soluble micronutrients, serum was extracted with hexane in the presence of butylated hydroxytoluene and the internal standard, Tocol. The extract was analyzed by HPLC using both visible $(450 \mathrm{~nm})$ and electrochemical detection with an ESA 4-channel detector set at 310, 390 and $470 \mathrm{mV}$ (ESA Biosciences, Chelmsford, MA), as described previously [46]. Total carotenoids were calculated as the sum of $\alpha$ - and $\beta$-carotene, $\beta$-cryptoxanthin, lutein and zeaxanthin.

\section{Study Design}

Subjects were randomized across two diet groups in a block size of four using sealed envelopes containing diet arm assignment on a folded piece of paper. These were opened sequentially at the baseline appointment after the baseline assessments were completed. All participants received written materials on diet and exercise, a pedometer (Omron HJ112, Omron Healthcare, Inc., Bannockburn, Illinois), and a bimonthly study newsletter providing tips for healthy eating and exercise to promote continued interest in the study. The pedometers were calibrated to the each participant's stride during the baseline study visit, and a handout on pedometer use was provided to all subjects.

\section{Control Arm}

Participants randomized to the control arm received the "My Pyramid" plan from the USDA that includes recommendations for daily exercise $(30 \mathrm{~min} /$ day $)$ and the Dietary Guidelines for Americans 2005 ( 2 cups fruits, 21/2 cups vegetables, moderate fat intake, 3 servings whole grains, and 3 servings low-fat dairy for a $2000 \mathrm{kcal}$ diet). They also received "Choices for Good Health" and "Cooking Smart" brochures from the American Cancer Society (revision 07/06 for both), and the afore-mentioned pedo-meters and study newsletters. These materials represent self-help information that an individual could reasonably obtain commercially or from the web. There was no dietary or exercise counseling provided, although written information was provided on how to correct any major nutritional inadequacies if uncovered. Subjects were free to make dietary and exercise changes on their own, if they so chose.

\section{Intervention Arm}

Subjects randomized to the intervention arm received written educational materials and telephone counseling from a registered dietitian trained in motivational interviewing (MI) techniques. The telephone counseling approach blended MI with social cognitive theory. Such a telephone-based approach would be useful for larger studies with study participants at multiple sites.

The written materials in this study arm included information regarding the rationale for following a high fruitvegetable/low-fat diet and weight control. They also received pedometers, a Daily Food and Exercise Log, a Fast Tracker, Fast Food Booklet, a Fat gram counter/food exchange list book (and 1-page, laminated, "frig list" summary of exchanges) and example menus at individually appropriate calorie levels. Fruit and vegetable and fat-gram goals were based on Total Energy Expenditure (TEE) in kcal/day as estimated from age, height, weight and physical activity using a published formula [47]. Fruit and vegetables goals were 7/day for less than $1700 \mathrm{kcal} /$ day, 8/day for $1700-1900$ $\mathrm{kcal} /$ day and 8/day for more than $1900 \mathrm{kcal} /$ day. Fat gram goals were calculated using $15 \%$ of calories from fat as the goal. Example 7-day menus were provided. The physical activity goal was to include least 30 minutes/day of moderate or more vigorous activity.

Initially subjects were asked to complete the Daily Food and Exercise Log which entailed writing down all foods eaten and minutes of physical activity. Once subjects became adept at enumerating servings of fruits and vegetables and fat grams, they could use the Fast Tracker that only tallied the goal food items plus minutes of moderate to vigorous physical activity. The Tracker was a modified version of that previously developed, and subjects were asked to fill out the 7-day tracker every other week within the first 6 months and one week per month for the last 6 months of the study [48]. The counseling plan was for the dietitian to contact subjects weekly for the first two calls, biweekly for the next 5 months and monthly for the last 6 months, for a total of 19 calls. All calls were tracked to determine frequency and length of contact. The self-monitoring logs were reviewed during the calls. Participants were permitted to contact the dietitian in between calls for additional counseling if they so desired.

The counseling approach combined principles of social cognitive theory and motivational interviewing (MI). MI approaches ask clients to take a more active role in the counseling, deliberating the relevance and benefits of lifestyle changes within their own situation. Existing MI techniques for dietary change were adapted for this study [49-52]. Subjects were involved in deriving their own shortterm goals and evaluating their progress towards goals. To 
build self-efficacy, any positive changes on the self-monitoring sheets were identified and praised. The counseling calls were recorded at 2 weeks, 5 months, and 11 months to verify counseling fidelity.

\section{Statistical Methods}

All data were entered into an Excel spreadsheet, checked for accuracy using descriptive statistics, and exported into SPSS version 17.0 for analysis. Comparisons of baseline variables by diet arm were done using two-sample t-tests and chi-square tests for continuous and categorical characteristics, respectively. Similar analyses were conducted for comparing the characteristics of women who did or did not complete 12 months on study (Table 1). Quality of life measures at baseline were compared between the groups that had initiated chemotherapy and the group that had not using two-sample t-tests.

Changes over time by the study arms for various measures of lifestyle, quality of life, health status and nutrients were evaluated using mixed models ANOVA resulting in an intention to treat analysis (Table 2). The different measures appearing in the table are treated as separate outcomes in the mixed model with time (baseline, 6 month and 12 month), group (intervention, control), and group-time interaction terms were used as factors. All models were further adjusted for chemotherapy initiation as that seemed to be associated with dropout. The clustering within subject was incorporated through a repeated measures analysis across time with the robust choice of an unstructured variance covariance matrix. Post-hoc pairwise comparisons across time within each study arm was carried out with a Bonferroni adjustment for multiple comparisons. Due to the non-normal nature of the outcome for various blood and other measures, a systematic investigation was carried out by means of a Box-Cox family of power transformations [53]. The transformations used to normalize the data were: logarithmic for weight, alpha-tocopherol, CRP, leptin, and insulin; inverse for waist circumference, and BMI; and square root for total carotenoids and gammatocopherol. These transformations made a normal error assumption tenable. The statistical inference was carried out

Table 1. Characteristics of 40 Study Subjects at Baseline. Data is given by whether or Not a Subject Completed 12 Months on Study. The Values are Mean and SD or Number and Percentage of Women in each Category

\begin{tabular}{|c|c|c|c|}
\hline Characteristic & Non-Completers $n=10$ & Completers $\mathbf{n}=\mathbf{3 0}$ & P-value ${ }^{a}$ \\
\hline Age (yrs) & $52.0(6.9)$ & $52.3(9.5)$ & 0.924 \\
\hline Body Fat (\%) & $41.6(8.9)$ & $38.3(6.8)$ & 0.239 \\
\hline Stage 1 & $1(10 \%)$ & $6(20 \%)$ & 0.690 \\
\hline Stage 3 & $2(20 \%)$ & $8(26.7 \%)$ & \\
\hline Mastectomy & $5(50 \%)$ & $16(53.4 \%)$ & 0.390 \\
\hline Already started chemotherapy & $8(80 \%)$ & $13(43.3 \%)$ & 0.069 \\
\hline TV hours/day & $2.2(1.3)$ & $1.6(1.0)$ & 0.159 \\
\hline FACT-B & $100(20)$ & $108(12)$ & 0.155 \\
\hline Physically active pre-diagnosis & $6(60 \%)$ & $26(86.7 \%)$ & 0.089 \\
\hline Total physical activity (min/week) & $134.0(195.8)$ & $196.5(133.6)$ & 0.263 \\
\hline Moderate/vigorous activity (min/week) & $95.0(161.4)$ & $131.8(108)$ & 0.416 \\
\hline Fruit and vegetables (servings/day) & $1.90(1.06)$ & $4.74(2.80)$ & 0.004 \\
\hline Blood total carotenoids $(\mu \mathrm{g} / \mathrm{ml})$ & $0.86(0.5)$ & $1.4(0.7)$ & 0.026 \\
\hline Blood cholesterol (mg/dl) & $192.1(28)$ & $188.6(39.2)$ & 0.805 \\
\hline Leptin & $22.7(29.7)$ & $14.4(10.2)$ & 0.194 \\
\hline
\end{tabular}

${ }^{a}$ P-values for continuous, parametric variables (age, body fat, weight at age 18, confident, convinced, FACT-G, FACT-B, fruit and vegetables) and non-parametric variables (BMI, TV hours/day, physical activity, moderate/vigorous activity, blood cholesterol, blood carotenoids, glucose, insulin, CRP) were obtained from Independent t-tests. P-values for other categorical variables (already started chemotherapy, physically active pre-diagnosis, cancer stage, mastectomy) were from Fisher's exact test. 
Table 2. Changes Over Time in each Diet Arm. Data are the Mean and Standard Error

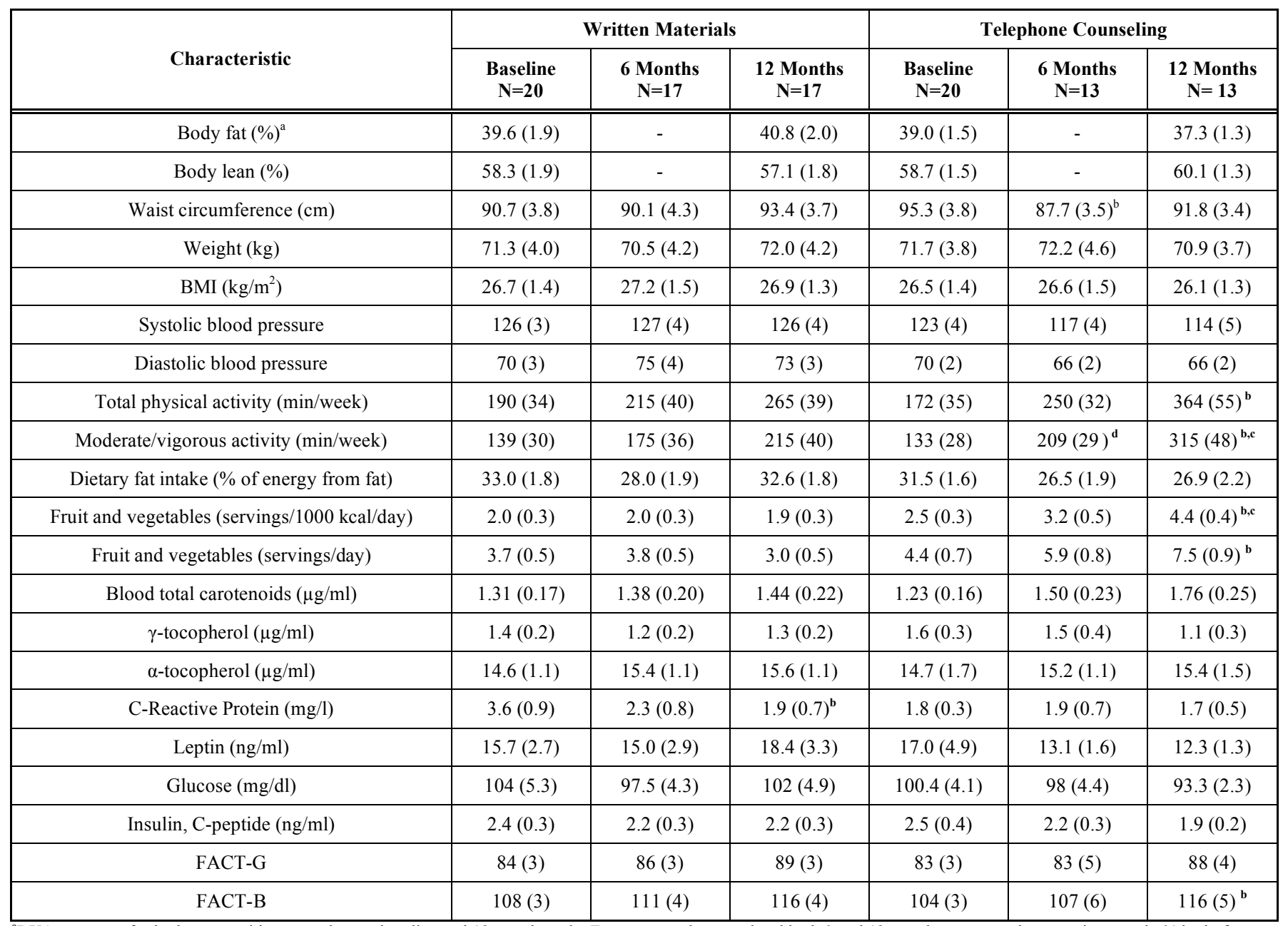

${ }^{a}$ DXA measures for body composition were done at baseline and 12 months only. For women who completed both 0 and 12 month measures, the mean increase in \% body fat over 12 months was 1.2 percentage points (SD 3.6, $\mathrm{n}=16$ ) in the written materials group (changing from a mean of 39.0 to $40.2 \%$ ) and -0.07 (SD 2.3 , $\mathrm{n}=13$ ) in the telephone group (changing from a mean of 37.4 to $37.3 \%$ ).

bignificantly different from baseline $(\mathrm{p}<0.05)$.

'Significantly different from 6 months $(\mathrm{p}<0.05)$.

${ }^{\mathrm{d}}$ Significantly different from baseline $(\mathrm{p}=0.05)$.

on the appropriately transformed measures. The responses to the evaluation questions were compared between the study arms using a two-sample Mann-Whitney-Wilcoxon test due to the non-normal data pattern (Table 3).

\section{RESULTS}

\section{Recruitment and Characteristics of Subjects at Baseline}

The number of new breast cancer patients presented at the Breast Care Forum for the months January through September, inclusive, was 464 . This number includes consults as well as patients who were treated at the University of Michigan Breast Care Clinic. During that time, 201 women enrolled in any clinical trial (treatment or non-treatment trials). A total of 77 women were referred to this study by the clinical care staff as being potentially eligible. Only two potentially eligible women were not referred to the study because of timing issues. Of the 77 women referred and screened, 71 were eligible and 40 women enrolled $(55 \%$ of those eligible).
Subjects were mainly European American ( $\mathrm{n}=35$, of which 2 were Hispanic); Asian (2), African American (2) and 1 of mixed heritage (Native American/European American). The subjects are all women with stage I-IIIA breast cancer and none had inflammatory breast cancer ( 30 ductal, 6 lobular and 4 mixed). The breast cancer stage distribution was: 7 with Stage I, 25 with Stage II and 8 with Stage III. Twenty women had already started chemotherapy when they enrolled in the study. A total of 30 women remained on study for the full 12 months (75\% retention). One Hispanic, one African American and 8 European American women did not complete 12 months on study (Fig. 1).

Eligible women were either scheduled for chemotherapy or no more than two weeks past starting chemotherapy. There was one instance where this time period was inadvertently three weeks for a subject who was treated at a different medical center three hours away. All but seven women received a combination of doxorubicin, cyclophosphamide and a taxane (five received cyclophosphamide and a taxane and two received doxorubicin and cyclophosphamide). Ten 
Table 3. Study Evaluation Questionnaire for Women who Completed 12 Months of Participation in each Diet Arm. Data are Shown are the Mean Answer (SD) from a Likert-type Scale with 1= Very much, 2= Mostly, 3= Neutral, 4= Sometimes, and $5=$ Not Really

\begin{tabular}{|c|c|c|c|}
\hline Study Aspect & $\begin{array}{c}\text { Written Materials } \\
\qquad \mathbf{N}=\mathbf{1 7}\end{array}$ & $\begin{array}{l}\text { Telephone Counseling } \\
\qquad \mathrm{N}=13\end{array}$ & $\begin{array}{c}\text { P- } \\
\text { value }^{a}\end{array}$ \\
\hline Being in the study was helpful to me & $3.00(1.32)$ & $1.46(0.88)$ & 0.002 \\
\hline The study gave me support in other ways besides diet and exercise & $3.23(1.15)$ & $1.54(1.13)$ & 0.001 \\
\hline Being in the study was stressful & $4.47(1.12)$ & $4.46(1.13)$ & 0.837 \\
\hline Being in the study helped me improve my diet versus what I would have done on my own & $3.59(1.28)$ & $1.69(1.11)$ & $<0.001$ \\
\hline The newsletters were helpful & $2.75(1.24)$ & $1.92(1.19)$ & 0.075 \\
\hline I used the pedometer & $3.13(1.85)$ & $3.38(1.85)$ & 0.786 \\
\hline I am glad I was in the study & $2.0(1.15)$ & $1.38(0.87)$ & 0.121 \\
\hline
\end{tabular}

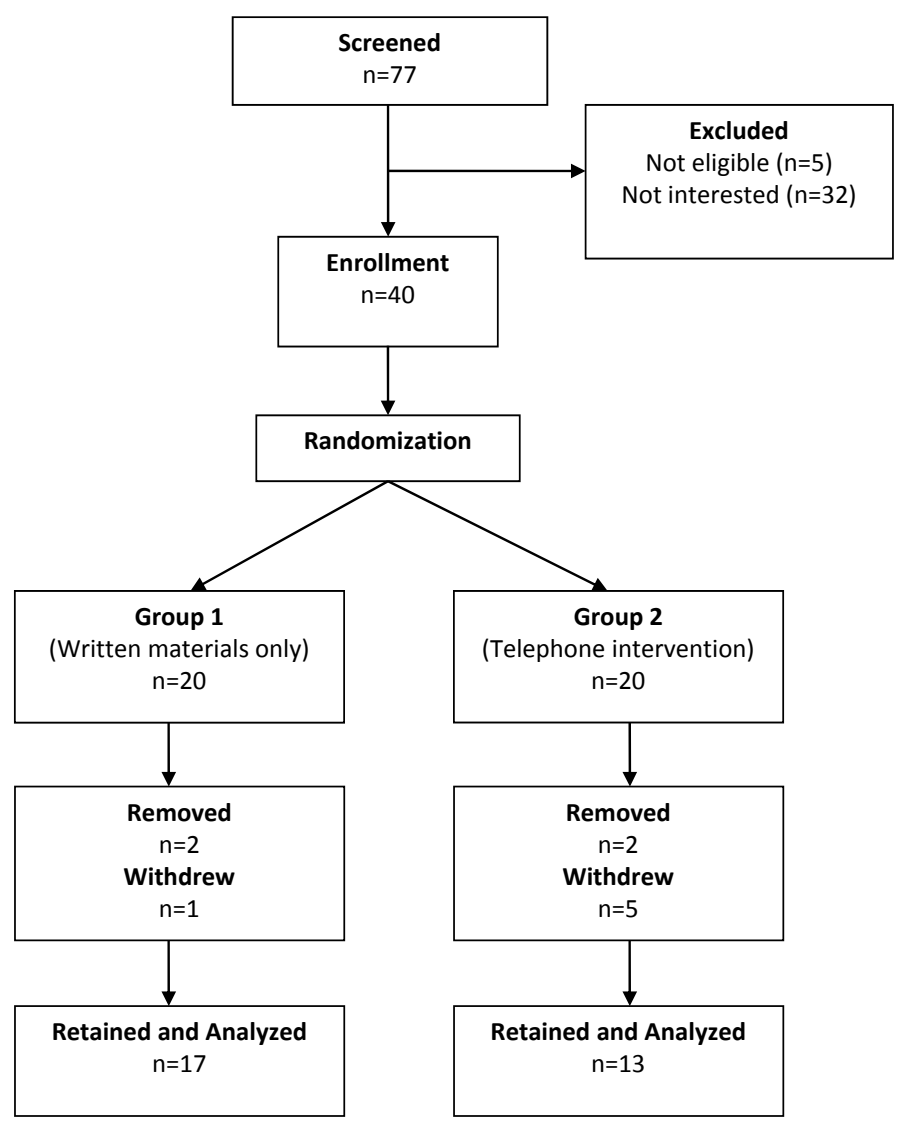

Fig. (1). Consolidated Standards of Reporting Trials (CONSORT) flow chart for recruitment and retention of study subjects in the Women's Healthy Lifestyles Study. Recruitment occurred January-September of 2008. Four women were removed from study due to serious medical issues.

women did not receive radiation therapy. All the subjects received steroidal medications. At 12 months, 13 were taking an aromatase inhibitor, eight were taking tamoxifen and five were taking trastuzumab.

Table 1 shows the demographic characteristics of women in the study by whether or not they completed 12 months on the study. The age range of enrolled women was 35-75 years and the BMI range was $18.2-42.9 \mathrm{~kg} / \mathrm{m}^{2}$. One woman had an eligible BMI of 18.5 at the eligibility determination and then had lost a few pounds before the baseline visit. Four of the women were removed from the study due to serious medical conditions, four indicated they had too much to do or were overwhelmed, and two gave no specific reason for withdrawing. In general, the women who completed the study had higher serving of fruit and vegetables, higher amount of total carotenoids in blood on an average than 
women who did not complete the study (Table 1). This may be an indication that perhaps women following relatively healthier lifestyles were more interested in participating in this study. There were similar trends for physical activity before diagnosis and glucose levels, with completers tending to be more active and to have lower glucose levels. The percentage of women who initiated chemotherapy before enrolling in the study was lower in completers.

In examining all the baseline study variables by diet arm assignment, women in the written materials arm had higher mean CRP levels than women in the telephone arm at baseline, due to several women with elevated levels (Table 2). All of the other clinical, behavioral and measured variables were not appreciably different between diet arms at baseline. The total time in active treatment (surgery, radiation, and/or chemotherapy) was a mean of 159 days for subjects in the written materials group and 167 days for the telephone group. The percentage of subjects at 12 months taking a hormonal treatment for breast cancer was $65 \%$ for the written materials group and $77 \%$ for the telephone group. Five women in each group who were premenopausal at study entry and all ten stopped menstruating by month 12 .

\section{Effects of Chemotherapy on Baseline Measures}

Half of the women in each arm had already started chemotherapy before the baseline visit. These subjects were evenly distributed in the two diet arms (10 subjects in each arm). We therefore examined whether this had any effects on the baseline blood and behavioral measures using a 2-sample t-test. Mean blood measures, anthropometric variables and dietary intakes of fat and fruits and vegetables did not differ significantly between women who had or had not started chemotherapy at baseline ( $\mathrm{p} \geq 0.1$ in each case). Certain aspects of well-being, however, were higher in the group that had not yet started chemotherapy, namely the physical (mean scores of 24 and 20, $\mathrm{p}=0.03$ ) and breast cancer-specific subscales (mean scores of 29 and 26, $\mathrm{p}=0.001$ ). This was not due to differences in the extent of surgery in the women who had or had not already started chemotherapy ( 7 of 20 versus 13 of 20, respectively, had a mastectomy). There were no differences in reported use of antidepressants in women who had or had not started chemotherapy at baseline. None of the other subscales nor the FACT-G score was significantly different in women who had one course of chemotherapy versus those that had not. Since the numbers of women who had already started chemotherapy were evenly distributed in the two diet groups, this was not used as a covariate in subsequent analyses.

\section{Compliance with the Intervention}

The mean number of counseling calls made in the telephone arm was 17.4, range 13-21, in the thirteen women who completed 12 months on study. This is close to the planned 19 calls. Extra calls were made when requested by a subject, and at other times a call was skipped due to scheduling issues with holidays, vacations, and personal matters that subjects needed to attend to. Self-monitoring logs of diet and exercise were turned in by subjects at a rate that was $70 \%$ of that requested, with only two women completing logs $<50 \%$ of the time on the requested occasions.
Subjects in the telephone arm made favorable changes in physical activity and in fruit and vegetable intakes that were statistically significant. Total physical activity increased to mean of 364 minutes/week and moderate/vigorous activity increased to a mean of 315 minutes/week at 12 months, slightly below the target of $350 \mathrm{~min} /$ week of moderate/ vigorous activity (Table 2). For fruit and vegetable intakes from un-announced recalls, the number of servings/day increased only in the telephone arm, and the mean reported intake at 12 months was just above the minimum intervenetion goal of 7 servings/day, not counting potatoes (Table 2). The increases in physical activity and fruit/vegetable intakes at 6 months were smaller than at 12 months. Multiple recalls would have been preferable, but we found previously that a single recall is comparable in capturing, energy, fat and fruit/vegetables intakes as other methods of assessment [54]. For fat intake, non-significant trends for decreasing fat intake were observed in the telephone arm as shown in Table 2.

Using the NCI fruit and vegetable screener, fruit and vegetable servings/day were higher than with the recalls (mean of $5.8 v s .4 .4$ servings/day at baseline by screener versus recalls, for all 40 subjects at baseline). In the telephone arm, intakes at 12 months were 9.8 by screener $v s .7 .3$ servings/day by recalls (data not shown). Recent literature confirms that the screener does over-estimate intakes [55]. Using the NCI fat screener, mean fat intake at baseline for all study subjects was $32.6 \%$ of energy, which is similar to that with recalls of $32.1 \%$, but change over time in the telephone arm by screener was smaller. In the telephone arm, fat intakes at 12 months were $31 \%$ by screener $v s .28 \%$ by recalls.

Pedometer usage was very low. All but one of the women in the telephone arm reported using the pedometers at least once, but only six of 13 women who completed the study reported using the pedometer more than three times. The six women who used the pedometer more than three times had roughly two-fold increased physical activity by questionnaire versus the seven women who used the pedometer less than three times, with the respective mean changes at 12 months as follows: moderate and vigorous physical activity (255 vs. 104 minutes/week), and total physical activity (212 vs. 75 minutes/week).

\section{Anthropometric Outcomes}

This pilot study was not powered to detect changes in anthropometric outcomes but beneficial trends were observed in the telephone arm. Body fat, body weight and waist circumference tended to decrease in the telephone arm and increase in the written materials arm (Table 2). Blood pressure also changed in a relatively more favorable direction in the telephone arm. In evaluating whether or not baseline BMI affected body fat change while on study, the results indicated that women in the written materials arm who had lower BMI at baseline tended to gain the most body fat during treatment for breast cancer (Fig. 2). The larger increases in body fat observed in the written arm were absent in the telephone arm (Fig. 2). In the written material arm, women with a baseline BMI of less than $30 \mathrm{~kg} / \mathrm{m}^{2}$ had a mean weight gain of $2.44 \mathrm{~kg}$ and in women with a BMI 


\section{Written Materials Group}

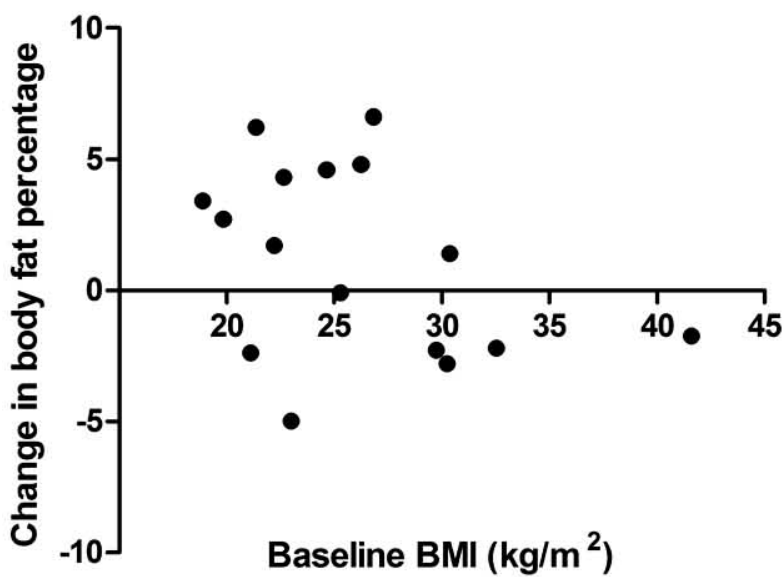

Telephone Counseling Group

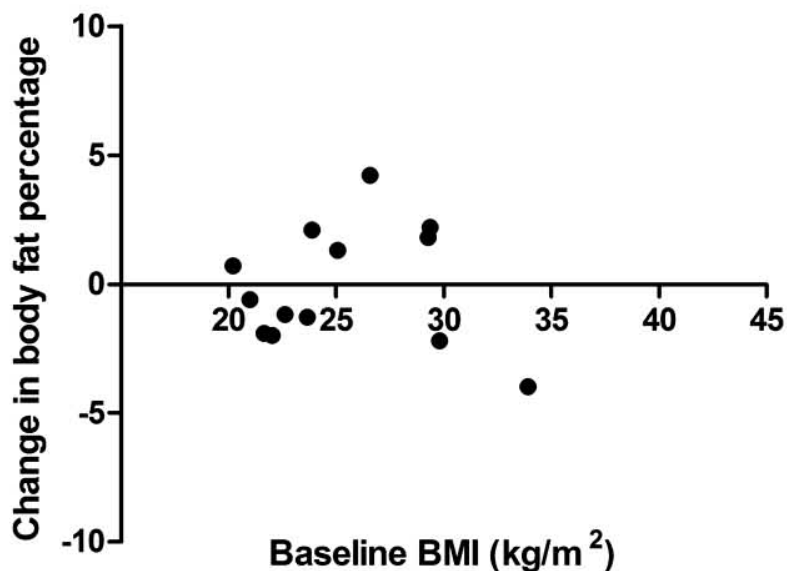

Fig. (2). Change in body fat over one year by baseline BMI in both diet groups. The absolute differences in body fat percentage between 12 months and baseline are shown.

greater than 30 mean weight change was $-1.53 \mathrm{~kg} / \mathrm{m}^{2}$ $(\mathrm{p}=0.010)$.

In evaluating which counseling variables were most closely associated with a decrease in body fat in the telephone arm, correlations of body fat change with the counseling and behavioral variables were explored. There was a trend for more minutes being spent counseling women who were less successful in controlling weight gain. These women likely needed more support than women who were more successful in meeting diet and exercise goals. There was also a trend for a greater decrease in body fat for women who completed a greater percentage of self-monitoring records (data not shown).

\section{Changes in Well-Being}

The mean FACT-B scores for women in this study at baseline shown in Table 2 were similar to that reported by Brady et al. for women undergoing breast cancer treatment [56]. Over 12 months of study, mean FACT-B scores improved in both study arms, but only significantly so in the telephone arm (Table 2). Mean time in active treatment (surgery, radiation, chemotherapy) was 163 days, or just over 5 months, indicating that many women were done with active treatment by 6 months on study and any intervention effects during treatment were therefore not captured.

\section{Changes in Blood Measures}

A number of blood measures were evaluated in this study. Serum carotenoids served as an unbiased measure of fruit and vegetable intakes. Since the number of subjects in the study was small, total carotenoids and not individual carotenoids were analyzed statistically. The increase in mean carotenoid levels in the telephone arm was greater than in the written materials arm, but this was not statistically significant (Table 2). Levels of tocopherols did not change significantly in either arm, but mean levels of $\gamma$-tocopherol did decrease in the telephone arm, which has been noted previously in women who follow low-fat diets [57]. Changes in insulin, glucose, and leptin did not change significantly although favorable changes were noted in the telephone arm
(Table 2). CRP decreased significantly in the written arm, but this was likely due to a high mean level at baseline in the written arm, which differed significantly from that in the telephone arm at baseline as mentioned previously.

\section{Study and Counseling Evaluations}

At the end of 12 months, study subjects who were retained evaluated the study using a questionnaire and this data is shown in Table 3. Neither group found that being in the study was stressful or difficult, and overall they were glad to be in the study. Women in the telephone arm reported that being in the study was helpful (giving scores of either 1 (very much) or 2 (mostly). Women in the written arm were neutral about whether or not being in the study was helpful to them (score of 3 ).

Women in the telephone group were also asked to evaluate the counseling that they received from the study dietitian. This questionnaire had 9 items and used a 5-point Likert-type scale for replies. All of the subjects who finished 12 months in the telephone arm thought that "The counselor listened to me" and that "The counselor was supportive and encouraging" all of the time. For four other items, all the subjects answered with either "all of the time" or "most of the time" and these were: 1) "The counselor asked my opinion about things", 2) "The counselor helped me think about why a low-fat, high fruit-vegetable diet is important", 3) "The counselor helped me think about why being physically active is important", and 4) "The study helped me follow a low-fat, high fruit-vegetable diet". For two additional items, all but one of the subjects answered with either "always" or "most of the time" and those items were: "The counselor discussed values that were important to me" and "The study helped me stay active". The one subject who did not agree answered those items with "not really" and "sometimes", respectively. Finally, for the item "The counselor asked permission before giving me information or advice", two women answered with "half the time" while the rest answered with "always" or "most of the time". The items on listening by the counselor, discussing values and asking permission reflect reasonably good adherence to principles of motivational interviewing in this study [58]. 


\section{DISCUSSION}

There was interest in breast cancer patients for joining a study focused on diet and physical activity during breast cancer treatment. Recruitment to this study was good with $55 \%$ of eligible women agreeing to participate (Fig. 1). In large dietary intervention studies of breast cancer survivors who were farther out from initial diagnosis than those in our study, participation of eligible women was similar, ranging $44-65 \%[21,37]$. For physical activity interventions, the largest reported trial instituted at the initiation of chemotherapy had an enrollment rate of $33 \%$ of eligible patients, which is still a substantial number [59]. Only one other trial thus far has reported a combined diet and exercise intervention during chemotherapy for breast cancer, and $81 \%$ of approached women were successfully enrolled in that 6month trial [8]. It is therefore feasible to enroll breast cancer patients in a diet and/or exercise trial during treatment with chemotherapy.

Unfortunately, the non-completion rate in our study was $25 \%$, with four women being removed from study due to medical issues and six women deciding to withdraw (Fig. 1). The withdrawal rate was higher in the telephone $(n=5)$ than the written material group $(\mathrm{n}=1)$. This somewhat higher than in our previous, year-long dietary intervention studies with breast cancer survivors past treatment or high risk women that did not have an exercise component, and those had attrition rates of about $20 \%[48,60]$. The women who did not complete this study tended to be heavier, less physically active, and had significantly lower fruit and vegetable intakes than women who did complete 12 months of study participation (Table 1). This indicates that women who were following relatively healthier lifestyles at baseline were perhaps more interested in complying with the study goals.

Compliance with the telephone counseling was good overall, with 17 of the planned 19 contacts being completed over 12 months, and $70 \%$ of the requested self-monitoring logs were returned. Relative to group-based and media-based interventions, individualized counseling can likely more fully address idiosyncratic psycho-social circumstances that affect weight control such as social support, coping skills, stress and self-efficacy. A possible additional benefit is the use of motivational interviewing (MI), especially during cancer treatment. With MI, subjects are more involved in determining how much information they would like to receive at any given point in time and how much they can realistically accomplish [58].

Compliance to fruit and vegetable goals and physical activity in this study was reasonably good but decreases in fat intake were more elusive (Table 2). A low-fat diet has been shown to decrease breast cancer recurrence in the Women's Intervention Nutrition Study (WINS) [21]. The mechanism involved may be via weight loss, or via a decrease in $\mathrm{n} 6$ fatty acid intakes since n6 fatty acids have strong promoting effects on mammary tumors in animal models [61]. The WINS study was initiated in 1987 when the concept of low-fat diets was quite novel. In our previous dietary intervention study in women at increased breast cancer risk that started in 1997, there was a high drop-out rate from the low-fat diet arm [57]. If decreasing n6 fatty acid intake is of key importance, other approaches could be tried, such as Mediterranean diets, which may have a better acceptance rate. Mediterranean diets also are linked with better control of body weight and abdominal adiposity than Western diets [62, 63].

For achieving the physical activity goal, counseling alone without use of pedometers appeared to be effective in our study since pedometer use was low. This could be due to the fact that participants were given the choice of either logging step counts or minutes of activity on the self-monitoring forms. The literature indicates that logging step count is critical for consistent pedometer use [64]. The present intervention was simple, it used no exercise equipment, facility or videos, and it was significantly effective for increasing physical activity over 12 months (Table 2).

This was a pilot study and it did not have power to detect changes in body fat, but body fat did increase in the written materials arm. The increase of about 2 percentage points over one year is generally similar to that observed in other small studies in which body fat was measured [8-11]. Large increases in body fat were not observed in the telephone counseling arm, consistent with the study goal of preventing body fat gain. Interestingly, the women who gained the most weight were not those with the highest BMI values at baseline (Fig. 2). This indicates that a program of healthy eating and physical activity would be beneficial to all women starting chemotherapy, not just overweight or obese women. Women participating in the telephone arm were much more positive about study participation than women in the written materials arm, and the telephone intervention was apparently helpful in many ways (Table $\mathbf{3}$ ).

\section{CONCLUSIONS}

This pilot study indicates that breast cancer patients were willing to comply with counseling for healthy diets and physical activity during cancer treatment. This lifestyle counseling can be used to prevent a gain in body fat during treatment. In the absence of counseling, body fat gain was greatest in women with a lower BMI at the start of treatment. Such a lifestyle approach should be applicable for improving usual care of all breast cancer patients, not just overweight or obese patients, to maximize health and well-being in survivorship.

\section{ACKNOWLEDGEMENTS}

We thank all the women who volunteered to participate in this study. We thank Mary Rapai, M.A., for assisting with study recruitment and follow-up and Patrick Brown for assisting with statistical analyses. The study was supported by the University of Michigan Metabolomics and Obesity Center (grant DK 089503), the Chemistry Core of the Michigan Diabetes Research and Training Center (grant DK020572), the Michigan Clinical Research Unit (grant UL1 RR024986), the University of Michigan Comprehensive Cancer Center Core Grant (grant P30 CA46592) and the Barbara Padnos Research Fund. The contents of this article are solely the responsibility of the authors and do not necessarily represent the official views of NCRR or NIH. 


\section{TRIAL REGISTRATION}

Clinical Trials registration NCT00583726.

\section{ABBBREVIATIONS}

BMI $=$ Body mass index

$\mathrm{CRP}=\mathrm{C}$-reactive protein

DXA $=$ Dual-energy X-ray absorptiometry

FACT-B $=$ Functional Assessment of Cancer Therapy Breast

SD $\quad=$ Standard deviation

\section{REFERENCES}

[1] Rowland J, Mariotto A, Aziz N, et al. Cancer Survivorship - United States, 1971--2001. Morbidity and Mortality Weekly Report 2004; 53: 526-9.

[2] Chlebowski RT, Aiello E, McTiernan A. Weight loss in breast cancer patient management. J Clin Oncol 2002; 20: 1128-43.

[3] Ballard-Barbash R, Hunsberger S, Alciati MH, et al. Physical activity, weight control, and breast cancer risk and survival: clinical trial rationale and design considerations. J Natl Cancer Inst 2009; 101: 630-43

[4] Sestak I, Distler W, Forbes JF, Dowsett M, Howell A, Cuzick J. Effect of body mass index on recurrences in tamoxifen and anastrozole treated women: an exploratory analysis from the ATAC trial. J Clin Oncol 2010; 28: 3411-5.

[5] Nichols HB, Trentham-Dietz A, Egan KM, et al. Body mass index before and after breast cancer diagnosis: associations with allcause, breast cancer, and cardiovascular disease mortality. Cancer Epidemiol Biomarkers Prev 2009; 18: 1403-9.

[6] Saquib N, Flatt SW, Natarajan L, et al. Weight gain and recovery of pre-cancer weight after breast cancer treatments: evidence from the women's healthy eating and living (WHEL) study. Breast Cancer Res Treat 2007; 105: 177-86.

[7] Kroenke CH, Chen WY, Rosner B, Holmes MD. Weight, weight gain, and survival after breast cancer diagnosis. J Clin Oncol 2005; 23: $1370-8$.

[8] Demark-Wahnefried W, Case LD, Blackwell K, et al. Results of a diet/exercise feasibility trial to prevent adverse body composition change in breast cancer patients on adjuvant chemotherapy. Clin Breast Cancer 2008; 8: 70-9.

[9] Campbell KL, Lane K, Martin AD, Gelmon KA, McKenzie DC. Resting energy expenditure and body mass changes in women during adjuvant chemotherapy for breast cancer. Cancer Nurs 2007; 30: 95-100

[10] Freedman RJ, Aziz N, Albanes D, et al. Weight and body composition changes during and after adjuvant chemotherapy in women with breast cancer. J Clin Endocrinol Metab 2004; 89: 2248-53.

[11] Harvie MN, Campbell IT, Baildam A, Howell A. Energy balance in early breast cancer patients receiving adjuvant chemotherapy. Breast Cancer Res Treat 2004; 83: 201-10.

[12] Lahmann PH, Lissner L, Berglund G. Breast cancer risk in overweight postmenopausal women. Cancer Epidemiol Biomarkers Prev 2004; 13: 1414.

[13] Wing RR, Hill JO. Successful weight loss maintenance. Annu Rev Nutr 2001; 21: 323-41.

[14] Schulze MB, Fung TT, Manson JE, Willett WC, Hu FB. Dietary patterns and changes in body weight in women. Obesity (Silver Spring) 2006; 14: 1444-53.

[15] Newby PK, Weismayer C, Akesson A, Tucker KL, Wolk A. Longitudinal changes in food patterns predict changes in weight and body mass index and the effects are greatest in obese women. J Nutr 2006; 136: 2580-7.

[16] Baxter AJ, Coyne T, McClintock C. Dietary patterns and metabolic syndrome--a review of epidemiologic evidence. Asia Pac J Clin Nutr 2006; 15: 134-42.
Fung TT, Willett WC, Stampfer MJ, Manson JE, Hu FB. Dietary patterns and the risk of coronary heart disease in women. Arch Intern Med 2001; 161: 1857-62.

[18] Byers T, Sedjo RL. A weight loss trial for breast cancer recurrence: pre-menopausal, post-menopausal, both, or neither? Cancer Causes Control 2006; 17: 1-3.

[19] Schairer C, Mink PJ, Carroll L, Devesa SS. Probabilities of death from breast cancer and other causes among female breast cancer patients. J Natl Cancer Inst 2004; 96: 1311-21.

[20] Herman DR, Ganz PA, Petersen L, Greendale GA. Obesity and cardiovascular risk factors in younger breast cancer survivors: The Cancer and Menopause Study (CAMS). Breast Cancer Res Treat 2005; 93: 13-23.

[21] Chlebowski RT, Blackburn GL, Thomson CA, et al. Dietary fat reduction and breast cancer outcome: interim efficacy results from the Women's Intervention Nutrition Study. J Natl Cancer Inst 2006; 98: 1767-76.

[22] Pierce JP, Stefanick ML, Flatt SW, et al. Greater survival after breast cancer in physically active women with high vegetable-fruit intake regardless of obesity. J Clin Oncol 2007; 25: 2345-51.

[23] Saris WH, Blair SN, van Baak MA, et al. How much physical activity is enough to prevent unhealthy weight gain? Outcome of the IASO 1st Stock Conference and consensus statement. Obes Rev 2003; 4:101-14.

[24] Jakicic JM, Otto AD. Treatment and prevention of obesity: what is the role of exercise? Nutr Rev 2006; 64: S57-61.

[25] Haskell WL, Lee IM, Pate RR, et al. Physical activity and public health: updated recommendation for adults from the American College of Sports Medicine and the American Heart Association. Med Sci Sports Exerc 2007; 39: 1423-34.

[26] Phelan S, Roberts M, Lang W, Wing RR. Empirical evaluation of physical activity recommendations for weight control in women. Med Sci Sports Exerc 2007; 39: 1832-6

[27] Visovsky C. Muscle strength, body composition, and physical activity in women receiving chemotherapy for breast cancer. Integr Cancer Ther 2006; 5: 183-91.

[28] Markes M, Brockow T, Resch KL. Exercise for women receiving adjuvant therapy for breast cancer. Cochrane Database Syst Rev 2006: CD005001.

[29] McTiernan A, Ulrich C, Kumai C, et al. Anthropometric and hormone effects of an eight-week exercise-diet intervention in breast cancer patients: results of a pilot study. Cancer Epidemiol Biomark Prevent 1998; 7: 477-81.

[30] Courneya KS. Exercise in cancer survivors: an overview of research. Med Sci Sports Exerc 2003; 35: 1846-52.

[31] Ingram C, Courneya KS, Kingston D. The effects of exercise on body weight and composition in breast cancer survivors: an integrative systematic review. Oncol Nurs Forum 2006; 33: 937-47.

[32] Darga LL, Magnan M, Mood D, Hryniuk WM, DiLaura NM, Djuric Z. Quality of life as a predictor of weight loss in obese breast cancer survivors. Oncol Nursing Forum 2007; 34: 86-92.

[33] Windsor PM, Potter J, McAdam K, McCowan C. Evaluation of a fatigue initiative: information on exercise for patients receiving cancer treatment. Clin Oncol (R Coll Radiol) 2009; 21: 473-82.

[34] Payne JK, Held J, Thorpe J, Shaw H. Effect of exercise on biomarkers, fatigue, sleep disturbances, and depressive symptoms in older women with breast cancer receiving hormonal therapy. Oncol Nurs Forum 2008; 35: 635-42.

[35] McInnes JA, Knobf MT. Weight gain and quality of life in women treated with adjuvant chemotherapy for early-stage breast cancer. Oncol Nurs Forum 2001; 28: 675-84.

[36] Kolotkin RL, Crosby RD, Williams GR. Health-related quality of life varies among obese subgroups. Obes Res 2002; 10: 748-56.

[37] Johnson-Kozlow M, Rock CL, Gilpin EA, Hollenbach KA, Pierce JP. Validation of the WHI Brief Physical Activity Questionnaire among Women Diagnosed with Breast Cancer. Am J Health Behav 2007; 31: 193-202.

[38] Cella D. Factors influencing quality of life in cancer patients: anemia and fatigue. Semin Oncol 1998; 25: 43-6.

[39] Brady MJ, Cella DF, Mo F, et al. Reliability and validity of the Functional Assessment of Cancer Therapy-Breast quality-of-life instrument. J Clin Oncol 1997; 15: 974-86.

[40] Thompson FE, Midthune D, Subar AF, Kahle LL, Schatzkin A, Kipnis V. Performance of a short tool to assess dietary intakes of fruits and vegetables, percentage energy from fat and fibre. Public Health Nutr 2004; 7: 1097-105. 
[41] Thompson F, Kipnis V, Subar A, et al. Performance of a Short Instrument to Estimate Usual Dietary Intake of Percent Calories from Fat. Eur J Clin Nutr 1998; 52 (suppl 2): S63.

[42] Snyder DC, Sloane R, Lobach D, et al. Agreement between a brief mailed screener and an in-depth telephone survey: observations from the Fresh Start study. J Am Diet Assoc 2004; 104: 1593-6.

[43] Conway JM, Ingwersen LA, Moshfegh AJ. Accuracy of dietary recall using the USDA five-step multiple-pass method in men: an observational validation study. J Am Diet Assoc 2004; 104: 595603.

[44] Conway JM, Ingwersen LA, Vinyard BT, Moshfegh AJ. Effectiveness of the US Department of Agriculture 5-step multiplepass method in assessing food intake in obese and nonobese women. Am J Clin Nutr 2003; 77: 1171-8.

[45] Friedewald WT, Levy RJ, Fredrickson DS. Estimation of the concentration of low-density lipoprotein cholesterol in plasma without use of the preparative ultracentrifuge. Clin Chem 1972; 18 : 499-502.

[46] Djuric Z, Ren J, Blythe J, VanLoon G, Sen A. A Mediterranean dietary intervention in healthy American women changes plasma carotenoids and fatty acids in distinct clusters. Nutr Res 2009; 29: 156-63.

[47] Gerrior S, Juan W, Basiotis P. An easy approach to calculating estimated energy requirements. Prev Chronic Dis 2006; 3: A129.

[48] Djuric Z, Poore KM, Depper JB, et al. Adherence with low-fat and high fruit and vegetable diets in the Nutrition and Breast Health Study: effects on body weight. Nutr Cancer 2002; 43:141-51.

[49] Resnicow K, Jackson A, Wang T, Dudley W, Baranowski T. A motivational interviewing intervention to increase fruit and vegetable intake through black churches: results of the eat for life trial. Am J Public Health 2001; 91: 1686-93.

[50] Hecht J, Borrelli B, Breger RK, Defrancesco C, Ernst D, Resnicow $\mathrm{K}$. Motivational interviewing in community-based research: experiences from the field. Ann Behav Med 2005; 29 (Suppl): 2934.

[51] Resnicow K, Jackson A, Blissett D, et al. Results of the healthy body healthy spirit trial. Health Psychol 2005; 24: 339-48.

[52] Resnicow K, Taylor R, Baskin M, McCarty F. Results of go girls: a weight control program for overweight African-American adolescent females. Obes Res 2005; 13: 1739-48.
[53] Box GEP, Cox DR. An analysis of transformations. J Royal Statistic Soc Ser B 1964; 26: 211-52.

[54] Radakovich K, Heilbrun LK, Venkatranamamoorthy R, Lababidi S, Klurfeld DM, Djuric Z. Women participating in a dietary intervention trial maintain dietary changes without much effect on household members. Nutr Cancer 2006; 55: 44-52.

[55] Greene GW, Resnicow K, Thompson FE, et al. Correspondence of the NCI Fruit and Vegetable Screener to repeat 24-H recalls and serum carotenoids in behavioral intervention trials. J Nutr 2008; 138: 200S-4S.

[56] Brady MJ, Cella DF, Mo F, et al. Reliability and validity of the Functional Assessment of Cancer Therapy-Breast Quality-of-Life instrument. J Clin Oncol 1997; 15: 974-86.

[57] Djuric Z, Ren J, Mekhovich O, Venkatranamoorthy R, Heilbrun LK. Effects of high fruit-vegetable and/or low-fat intervention on plasma micronutrient levels. J Am Coll Nutr 2006; 25: 178-87.

[58] Resnicow K, Dilorio C, Soet JE, Ernst D, Borrelli B, Hecht J. Motivational interviewing in health promotion: it sounds like something is changing. Health Psychol 2002; 21: 444-51.

[59] Courneya KS, Segal RJ, Gelmon K, et al. Predictors of supervised exercise adherence during breast cancer chemotherapy. Med Sci Sports Exerc 2008; 40: 1180-7.

[60] Djuric Z, DiLaura NM, Jenkins I, et al. Combining weight-loss counseling with the Weight Watchers plan for obese breast cancer survivors. Obes Res 2002; 10: 657-65.

[61] Freedman LS, Clifford C, Messina M. Analysis of dietary fat, calories and body weight, and the development of mammary tumors in rats and mice: a review. Cancer Res 1990; 50: 5710-9.

[62] Brown T, Avenell A, Edmunds LD, et al. Systematic review of long-term lifestyle interventions to prevent weight gain and morbidity in adults. Obes Rev 2009; 10: 627-38.

[63] Romaguera $\mathrm{D}$, Norat $\mathrm{T}$, Mouw $\mathrm{T}$, et al. Adherence to the Mediterranean diet is associated with lower abdominal adiposity in European men and women. J Nutr 2009; 139: 1728-37.

[64] Bravata DM, Smith-Spangler C, Sundaram V, et al. Using pedometers to increase physical activity and improve health: a systematic review. JAMA 2007; 298: 2296-304.

(C) Djuric et al.; Licensee Bentham Open.

This is an open access article licensed under the terms of the Creative Commons Attribution Non-Commercial License (http://creativecommons.org/licenses/by$\mathrm{nc} / 3.0 /$ ), which permits unrestricted, non-commercial use, distribution and reproduction in any medium, provided the work is properly cited. 